\title{
Crystal structure of hexapraseodymium dititanium septasulfide hexaoxide, $\operatorname{Pr}_{6} \mathrm{Ti}_{2} \mathrm{~S}_{7} \mathrm{O}_{6}$
}

\author{
Anna S. Gardberg and James A. Ibers* \\ Northwestem University. Department of Chemistry, 2145 Sheridan Rd., Evanston, IL 60208-3113, USA
}

Received April 20. 2001, CSD-No. 409558

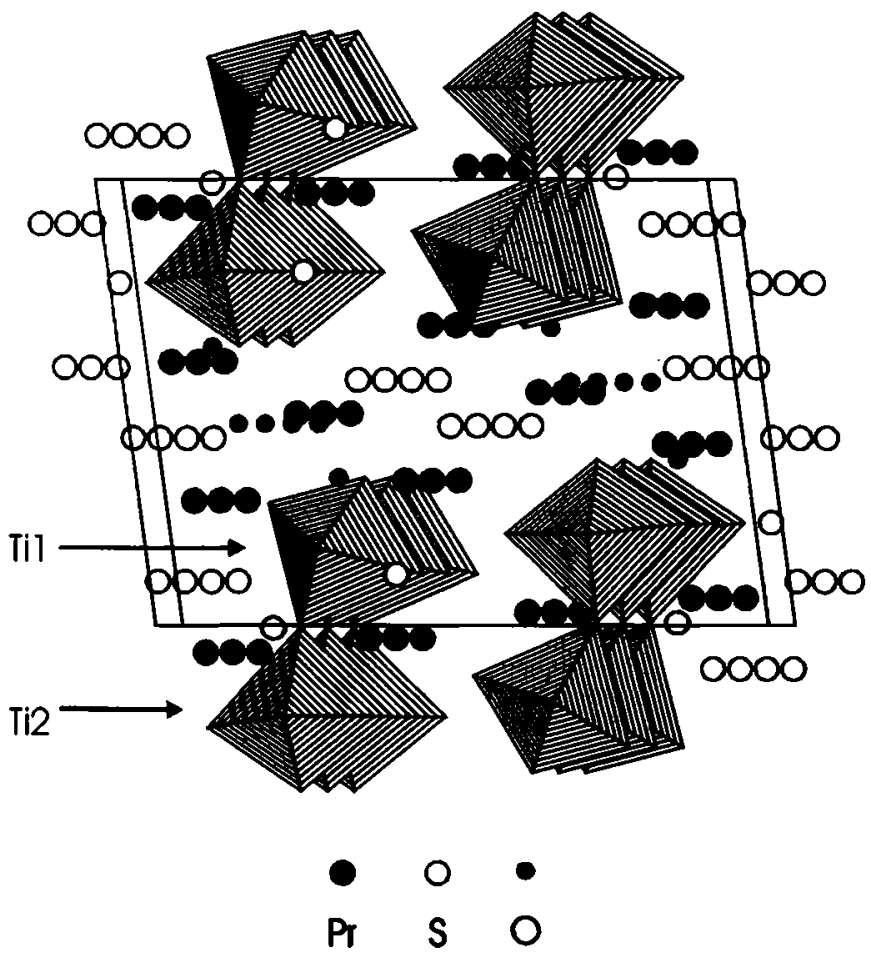

Abstract

$\mathrm{O}_{6} \mathrm{Pr}_{6} \mathrm{~S}_{7} \mathrm{Ti}_{2}$, monoclinic, $P 121 / m 1$ (No. 11), $a=11.944(2) \AA$, $b=3.9746(6) \AA, c=16.327(2) \AA, \beta=97.951(3)^{\circ}, V=767.6 \AA^{3}$, $Z=2, R_{\mathrm{gt}}(F)=0.036, w R_{\mathrm{ref}}\left(F^{2}\right)=0.086, T=153 \mathrm{~K}$.

\section{Source of material}

Single crystals of $\mathrm{P}_{6} \mathrm{Ti}_{2} \mathrm{~S}_{7} \mathrm{O}_{6}$ were prepared from the reaction of $\mathrm{Pr}_{2} \mathrm{~S}_{3}$ and $\mathrm{TiO}_{2}$ in 2:1 ratio. $\mathrm{KBr}$ flux was employed to encourage crystal growth. The reactants were ground together and loaded into a fused silica tube that was then evacuated to approximately $10^{-4}$ Torr and sealed. The tube was heated to $1223 \mathrm{~K}$ over 24 hours, kept there for eight days, cooled at $1 \mathrm{~K} / \mathrm{h}$ to $1123 \mathrm{~K}$, kept there for four days, and then cooled at $3 \mathrm{~K} / \mathrm{h}$ to room temperature. Black powder and lustrous red needles were obtained. The red crystals were found by EDS to contain $\mathrm{Pr}, \mathrm{Ti}$, and $\mathrm{S}$ in the approximate ratio $3: 1: 3$. The closely related compound $\mathrm{La}_{6} \mathrm{Ti}_{2} \mathrm{~S}_{8} \mathrm{O}_{5}$ was prepared previously [1] in an analogous manner from the reaction of $\mathrm{La}_{2} \mathrm{~S}_{3}$ and $\mathrm{TiO}_{2}$. In that preparation the tube was cooled at $3 \mathrm{~K} / \mathrm{h}$ directly from $1223 \mathrm{~K}$ to room temperature.

\begin{abstract}
Discussion
There are no bonding interactions among $\mathrm{S}$ and $\mathrm{O}$ atoms in $\mathrm{PT}_{6} \mathrm{Ti}_{2} \mathrm{~S}_{7} \mathrm{O}_{6}$ so the compound may be described formally as containing $\operatorname{Pr}(\mathrm{III})$ and $\mathrm{Ti}(\mathrm{IV})$. The unit cells of $\mathrm{P}_{6} \mathrm{Ti}_{2} \mathrm{~S}_{7} \mathrm{O}_{6}$ and $\mathrm{La}_{6} \mathrm{Ti}_{2} \mathrm{~S}_{8} \mathrm{O}_{5}(a=12.572(10) \AA, b=4.053(2) \AA, c=16.289(14) \AA$, $\left.\beta=96.79(1)^{\circ}\right)[1]$ are similar and there are some close resemblances between the two structures. For example, in both structures the $\mathrm{Til}$ and $\mathrm{Ti} 2$ atoms are in distorted octahedral environments. These octahedra form corner-sharing dimeric chains along [010] (see figure). However the bridging atom is an $\mathrm{S}$ atom in the present structure as opposed to an $\mathrm{O}$ atom in the $\mathrm{La}$ compound. The coordination environments of the $\mathrm{Ti}$ and $\mathrm{Ln}(\mathrm{Ln}=$ $\mathrm{Pr}, \mathrm{La}$ ) atoms in the two structures are often different. In the La compound, the predominant geometry about the six independent La atoms is capped trigonal prismatic whereas in the present Pr structure it is square antiprismatic. Of course, given the different stoichiometries the distribution of $\mathrm{O}$ and $\mathrm{S}$ atoms in these coordination polyhedra differs between the two structures. Distances in $\mathrm{Pr}_{6} \mathrm{Ti}_{2} \mathrm{~S}_{7} \mathrm{O}_{6}$ are consistent with those in other rare-earth oxysulfides: $d(\operatorname{Pr}-\mathrm{O})=2.337(5) \AA-2.955(14) \AA ; d(\mathrm{Pr}-\mathrm{S})=$ $2.838(2) \AA-3.192(3) \AA ; d(\mathrm{Ti}-\mathrm{O})=1.780(11) \AA-2.021(2) \AA$; $d(\mathrm{Ti}-\mathrm{S})=2.282(4) \AA-2.774(4) \AA$.
\end{abstract}

Table 1. Data collection and handling.
Crystal:

Wavelength:

$\mu$ :

Diffractometer, scan mode:

$2 \theta_{\max }$ :

$N(h k l)_{\text {measured, }} N(h k l)_{\text {unique: }}$

Criterion for $I_{\mathrm{obs}}, N(h k l)_{\mathrm{gt}}$ :

$N(\text { param })_{\text {refined: }}$

Program: red needle, size $0.012 \times 0.022 \times 0.160 \mathrm{~mm}$ Mo $K_{\alpha}$ radiation $(0.71073 \AA)$ $205.93 \mathrm{~cm}^{-1}$

Bruker SMART CCD, $\Delta \omega=0.3^{\circ}$

$57.64^{\circ}$

4997,2090

$I_{\text {obs }}>2 \sigma\left(I_{\text {obs }}\right), 1689$

115

SHELXTL [2]
Table 2. Atomic coordinates and displacement parameters (in $\AA^{2}$ ).

\begin{tabular}{llllll}
\hline Atom & Site & $x$ & $y$ & $z$ & $U_{\text {iso }}$ \\
\hline$O(1)$ & $2 e$ & $0.2052(7)$ & $1 / 4$ & $0.7792(6)$ & $0.012(2)$ \\
$O(2)$ & $2 e$ & $0.2750(9)$ & $1 / 4$ & $0.2231(7)$ & $0.027(3)$ \\
$O(3)$ & $2 e$ & $0.3326(7)$ & $1 / 4$ & $0.3868(5)$ & $0.010(2)$ \\
$O(6)$ & $2 e$ & $0.826(1)$ & $1 / 4$ & $0.6857(8)$ & $0.045(3)$
\end{tabular}

* Correspondence author (e-mail: ibers@chem.northwestern.edu) 
Table 3. Atomic coordinates and displacement parameters (in $\AA^{2}$ ).

\begin{tabular}{|c|c|c|c|c|c|c|c|c|c|c|}
\hline Atom & Site & $x$ & $y$ & $z$ & $U_{11}$ & $U_{22}$ & $U_{33}$ & $U_{12}$ & $U_{13}$ & $U_{23}$ \\
\hline $\operatorname{Pr}(1)$ & $2 e$ & $0.02967(6)$ & $1 / 4$ & $0.62158(5)$ & $0.0173(4)$ & $0.0122(4)$ & $0.0182(4)$ & 0 & $0.0094(3)$ & 0 \\
\hline $\operatorname{Pr}(2)$ & $2 e$ & $0.06186(6)$ & $1 / 4$ & $0.89181(5)$ & $0.0117(3)$ & $0.0097(4)$ & $0.0113(4)$ & 0 & $0.0047(3)$ & 0 \\
\hline $\operatorname{Pr}(3)$ & $2 e$ & $0.40749(6)$ & $1 / 4$ & $0.88264(4)$ & $0.0084(3)$ & $0.0099(4)$ & $0.0075(4)$ & 0 & $0.0017(3)$ & 0 \\
\hline $\operatorname{Pr}(4)$ & $2 e$ & $0.47591(6)$ & $1 / 4$ & $0.28938(4)$ & $0.0094(3)$ & $0.0101(4)$ & $0.0072(4)$ & 0 & $0.0020(3)$ & 0 \\
\hline $\operatorname{Pr}(5)$ & $2 e$ & $0.67449(6)$ & $1 / 4$ & $0.52643(4)$ & $0.0097(3)$ & $0.0103(4)$ & $0.0082(4)$ & 0 & $0.0013(3)$ & 0 \\
\hline $\operatorname{Pr}(6)$ & $2 e$ & $0.71887(6)$ & $1 / 4$ & $0.87687(5)$ & $0.0085(3)$ & $0.0106(4)$ & $0.0130(4)$ & 0 & $-0.0008(3)$ & 0 \\
\hline $\mathrm{Ti}(1)$ & $2 e$ & $0.2042(2)$ & $1 / 4$ & $0.3121(2)$ & $0.009(1)$ & $0.079(3)$ & $0.007(1)$ & 0 & $0.002(1)$ & 0 \\
\hline $\mathrm{Ti}(2)$ & $2 e$ & $0.7766(2)$ & $1 / 4$ & $0.2346(2)$ & $0.008(1)$ & $0.045(2)$ & $0.008(1)$ & 0 & $0.0015(9)$ & 0 \\
\hline$S(1)$ & $2 e$ & $0.0993(3)$ & $1 / 4$ & $0.0682(2)$ & $0.008(1)$ & $0.010(2)$ & $0.012(2)$ & 0 & $-0.001(1)$ & 0 \\
\hline$S(2)$ & $2 e$ & $0.1146(3)$ & $1 / 4$ & $0.4601(2)$ & $0.011(2)$ & $0.017(2)$ & $0.017(2)$ & 0 & $0.003(1)$ & 0 \\
\hline$S(3)$ & $2 e$ & $0.4212(2)$ & $1 / 4$ & $0.0603(2)$ & $0.011(2)$ & $0.012(2)$ & $0.006(2)$ & 0 & $0.003(1)$ & 0 \\
\hline$S(4)$ & $2 e$ & $0.4484(3)$ & $1 / 4$ & $0.5814(2)$ & $0.010(1)$ & $0.013(2)$ & $0.007(2)$ & 0 & $0.003(1)$ & 0 \\
\hline$S(5)$ & $2 e$ & $0.7689(2)$ & $1 / 4$ & $0.0717(2)$ & $0.008(1)$ & $0.011(2)$ & $0.010(2)$ & 0 & $-0.000(1)$ & 0 \\
\hline$S(6)$ & $2 e$ & $0.7936(3)$ & $1 / 4$ & $0.3756(2)$ & $0.012(2)$ & $0.026(2)$ & $0.008(2)$ & 0 & $0.001(1)$ & 0 \\
\hline$S(7)$ & $2 e$ & $0.9937(3)$ & $1 / 4$ & $0.2465(2)$ & $0.007(2)$ & $0.071(3)$ & $0.011(2)$ & 0 & $0.002(1)$ & 0 \\
\hline$O(4)$ & $2 e$ & $0.5469(7)$ & $1 / 4$ & $0.7864(6)$ & $0.014(4)$ & $0.013(5)$ & $0.006(5)$ & 0 & $0.000(3)$ & 0 \\
\hline$O(5)$ & $2 e$ & $0.6264(7)$ & $1 / 4$ & $0.2100(6)$ & $0.011(4)$ & $0.021(5)$ & $0.010(5)$ & 0 & $0.007(4)$ & 0 \\
\hline
\end{tabular}

Acknowledgments. This work was supported by the National Science Foundation (Grant DMR-0096676) and by the MRSEC program of the National Science Foundation (Grant DMR-0076097) at the Northwestern University Materials Research Center.

\section{References}

1. Cody, J. A.; Ibers, J. A.: Synthesis and Characterization of the New Rare-Earth/Transition-Metal Oxysulfides $\mathrm{La}_{6} \mathrm{Ti}_{2} \mathrm{~S}_{8} \mathrm{O}_{5}$ and $\mathrm{La}_{4} \mathrm{Ti}_{3} \mathrm{~S}_{4} \mathrm{O}_{8}$ J. Solid State Chem. B114 (1995) 406-412.

2. Sheldrick, G. M.: SHELXTL, DOS/Windows/NT Version 5.10., Bruker Analytical X-Ray Instruments, Inc., Madison, WI, USA 1998. 